\title{
PHASE DIAGRAM OF A CHARGED BOSE GAS *
}

\author{
J. P. HANSEN, B. JANCOVICI, and DANIEL SCHIFF \\ Laboratoire de Physique Théorique et Hautes Energies, Bâtiment 211, \\ Université Paris-Sud, Orsay, France
}

\begin{abstract}
The phase diagram of a charged Bose gas is drawn. The domains of existence of the solid, fluid and superfluid phases are discussed. It is predicted that superdense helium can be superfluid at densities higher than $10^{6} \mathrm{~g} \mathrm{~cm}^{-3}$.
\end{abstract}

* Presented by B. Jancovici. This paper has been published in Phys. Rev. Letters 29, 991 (1972). 